\title{
THE ABSCISSA OF ABSOLUTE SUMMABILITY OF LAPLACE INTEGRALS
}

\author{
GODFREY L. ISAACS ${ }^{1}$
}

ABSTRACT. With $A(u)$ of bounded variation over every finite interval of the nonnegative real axis, we write $C(w)=\int_{0}^{w} e^{-u s} d A(u)$ and (formally)

$$
R\left(k^{\prime}, w\right)=\left(\Gamma\left(k^{\prime}+1\right)\right)^{-1} \int_{w}^{\infty}(u-w)^{k^{\prime}} d A(u) \quad\left(k^{\prime} \geqq 0\right) .
$$

It is shown that if $k$ is positive and fractional and if $e^{-w s^{\prime}} R(k, w)$ is summable $|C, 0|$ for some $s^{\prime}$ whose real part is negative, then $C(w)$ is summable $|C, k+\varepsilon|$ for each $\varepsilon>0$, where $s$ is such that its real part is greater than that of $s^{\prime}$; if $k$ is nonnegative and integral the result holds with $\varepsilon=0$. Together with a 'converse' result, this may be used to show that if the abscissa of $|C, k|$ summability of $\int_{0}^{\infty} e^{-u s} d A(u)$ is negative then it equals

$$
\limsup _{w \rightarrow \infty} w^{-1} \log \int_{w}^{\infty}|d R(k, u)|
$$

for all $k \geqq 0$ except one fractional value.

1. If

$$
\Gamma(k+1) x^{-k} F_{k}(a ; x) \equiv x^{-k} \int_{a}^{x}(x-u)^{k} f(u) d A(u)=L+o(1)
$$

as $x \rightarrow \infty$ (or is of bounded variation over $[a, \infty$ ), with limit $L$ ), where $A(u)$ is of bounded variation over every finite interval of the nonnegative real axis and the integral is taken in the Riemann-Stieltjes sense, we say that $F(a ; x)$ (i.e., $\left.F_{0}(a ; x)\right)$ is summable $(C, k)($ or $|C, k|)$ to $L$. If now, $\left(\Gamma\left(k^{\prime}+1\right)\right)^{-1} \int_{w}^{x}(u-w)^{k^{\prime}} d A(u)$ is summable $(C, k)$ (or $\left.|C, k|\right)$ to $L$, we shall say that

$$
R\left(k^{\prime}, w\right) \equiv\left(\Gamma\left(k^{\prime}+1\right)\right)^{-1} \int_{w}^{\infty}(u-w)^{k^{\prime}} d A(u)
$$

exists in the $(C, k)($ or $|C, k|)$ sense and has value $L$. The notation $h(x)=$ $L+o(1)|C, k|$ will mean

$$
\int_{1}^{x} d h(u)=L-h(1)+o(1)|C, k| .
$$

Presented to the Society, August 2, 1969; received by the editors March 8, 1971. AMS 1970 subject classifications. Primary 40A10, 40F05, 40G05; Secondary 40D15. Key words and phrases. Laplace integral, summable $|C, k|$, abscissa of summability. ${ }^{1}$ Research supported in part by a CUNY Faculty Research grant (no. 1051).

(c) American Mathematical Society 1972 
We shall write $V$ for the class of functions of bounded variation over $[1, \infty) ;[k]$ for the largest integer less than or equal to $k,\langle k\rangle$ for $k-[k]$; $c, c_{r}, c_{r}^{\prime}$ for constants; and

$$
C_{k}(x)=(\Gamma(k+1))^{-1} \int_{0}^{x}(x-u)^{k} e^{-u s} d A(u) \quad(k \geqq 0), C(x)=C_{0}(x),
$$

where $s$ is complex and $\operatorname{Re}(s)=\sigma$. We have now,

THEOREM A [6]. If $k$ is positive and fractional, and if $C(w)$ is summable $|C, k|$ for some s such that $\sigma<0$, then $R(k+\delta, w)$ exists in the $|C, k|$ sense and

$$
e^{-w s} w^{-k} R(k+\delta, w)=o(1)|C, 0| \text { for each } \delta>0 .
$$

THEOREM B. If $k$ is positive and fractional, and if $R(k, w)$ exists in the $(C, p)$ sense for some $p \geqq k$ and satisfies that $e^{-w s^{\prime}} R(k, w)$ is summable $|C, 0|$ for some $s^{\prime}$ such that $\operatorname{Re}\left(s^{\prime}\right)=\sigma^{\prime}<0$, then $C(w)$ is summable $|C, k+\varepsilon|$ whenever $\sigma>\sigma^{\prime}$, for every $\varepsilon>0$.

Theorem B is proved below. For $k$ integral, by [3, Theorem 3] and an easy version of the proof below, we see that Theorems $A$ and $B$ are true with $\delta=0, \varepsilon=0$ respectively. The $(\mathrm{C})$ analogues in all cases are substantially given in [4]. For a general discussion of related results see [8, p. 10].

2. Proof of Theorem B. This will follow from

LEMMA 1. Under the hypotheses of Theorem $\mathrm{B}$, if $0 \leqq \varepsilon<1-\langle k\rangle$, then for $\sigma>\sigma^{\prime}$

where

$$
w^{-k-\varepsilon} C_{k+\varepsilon}(w)+(-1)^{[k]} w^{-k-\varepsilon} e^{-w s} U^{(\langle k\rangle+\varepsilon)}(w) \text { is in } V,
$$

$$
U^{(r)}(w)=(\Gamma(r))^{-1} \int_{w-1}^{w}(w-u)^{r-1} R([k], u) d u \quad(r>0) .
$$

LEMMA 2. Under the hypotheses of Theorem $\mathrm{B}$,

$$
e^{-w s^{\prime}} U^{\langle\langle k\rangle+\varepsilon)}(w) \text { is in } V \text { for each } \varepsilon>0 \text {. }
$$

Proof of Lemma 1. Case $\varepsilon=0$. We may take $A$ continuous on the right. Then by [5, Theorem 2], and [4, Lemma 2, Corollary],

$$
R([k], u)=(\Gamma(1-\langle k\rangle))^{-1}\left(\int_{u}^{u+1}+\int_{u+1}^{\infty}\right)(t-u)^{-\langle k\rangle} d R(k, t) ;
$$

we see that the integral of $R([k], u)$ from $w$ to $\infty$ is convergent, and hence by $[5$, p. 236 line -2$]$,

$$
R([k]+1, u) \text { exists in the }(C, p) \text { sense. }
$$


We now write
(a) $R(k, t)=e^{t s^{\prime}} f(t)$
(b) $R([k]+1, t)=e^{t s^{\prime}} g(t)$.

Then by hypothesis, $f(t)$ is in $V$. By [4, Lemma 2],

$$
R([k]+1, w)=(\Gamma(1-\langle k\rangle))^{-1} \int_{w}^{\infty}(t-w)^{-\langle k\rangle} R(k, t) d t,
$$

the integral being convergent by our hypothesis. Inserting (2)(a), then putting $t=w+x$ and applying [6, Lemma 2], we see that $\dot{g}(w)$ is in $V$. By $[4,(64)-(70)]$ we have

$$
C_{k}(w)=\sum_{r=0}^{[k]} c_{r} w^{k-r}+\sum_{r=0}^{[k]+1} c_{r}^{\prime} I_{r}=A+B,
$$

say, where

$$
I_{r}=\int_{0}^{w} R([k], u)(w-u)^{k-r} e^{-u s} d u .
$$

Writing $R([k], u)$ as the derivative of $-e^{u s^{\prime}} g(u)$ (by (1) and [4, Lemma 2, Corollary]) we now obtain

$$
w^{-k} I_{r}=-w^{-r}\left(s^{\prime} N_{1}(r, w)+N_{2}(r, w)\right) \quad(r=0,1, \cdots,[k])
$$

where

$$
N_{1}(r, w)=\int_{0}^{w}(1-u / w)^{k-r} e^{-u\left(s-s^{\prime}\right)} g(u) d u
$$

and $N_{2}(r, w)$ is the same integral with $g$ replaced by $g^{\prime}$. Also

$$
I_{[k]+1}=\left(\int_{0}^{w-1}+\int_{w-1}^{w}\right) R([k], u)(w-u)^{\langle k\rangle-1} e^{-u s} d u=P+Q,
$$

say. But

$$
P=-w^{\langle k\rangle-1}\left(s^{\prime} N_{1}([k]+1, w-1)+N_{2}([k]+1, w-1)\right)
$$

and $Q$ may be expressed as

$$
\begin{aligned}
e^{-w s} \int_{w-1}^{w} R([k], u)(w-u)^{\langle k\rangle-1} d u & \\
& +\int_{w-1}^{w} R([k], u)(w-u)^{\langle k\rangle-1}\left(e^{-u s}-e^{-w s}\right) d u=Q_{1}+Q_{2},
\end{aligned}
$$

say. An integration by parts of $Q_{2}$, followed by the insertion of (2)(b) 
and then the substitution $u=w-x$, gives

$$
\begin{aligned}
Q_{2}=( & \left.1-e^{-s}\right) e^{-(w-1)\left(s-s^{\prime}\right)} g(w-1)-s \int_{0}^{1} x^{\langle k\rangle-1} e^{-(w-x)\left(s-s^{\prime}\right)} g(w-x) d x \\
& -(\langle k\rangle-1) \int_{0}^{1} x^{\langle k\rangle-2}\left(1-e^{-s x}\right) e^{-(w-x)\left(s-s^{\prime}\right)} g(w-x) d x .
\end{aligned}
$$

Applying [4, Lemma 2] to the last two integrals, and also to those in (6) and (5), shows that $Q_{2}$ and $P$ are in $V$ and that $w^{-k} I_{r}$ is in $V$ $(r=0,1, \cdots,[k])$. Since $Q_{1}$ is just $\Gamma(\langle k\rangle) e^{-w s} U^{\langle\langle k\rangle)}(w)$ and (in (4)) $c_{[k]+1}^{\prime}=(-1)^{[k]+1}(\Gamma(\langle k\rangle))^{-1}$, this completes the proof.

Case $\varepsilon>0$. By (1) and [1, p. 300], $R(k+\varepsilon, w)$ certainly exists in the $(C, p)$ sense, and thus by [4, Lemma 2], (3) holds with $R([k]+1, w)$ and $-\langle k\rangle$ replaced by $R(k+\varepsilon, w)$ and $\varepsilon-1$ respectively. Hence $e^{-w s^{\prime}} R(k+\varepsilon, w)$ is in $V$. The required result now follows from the previous case with $k$ replaced by $k+\varepsilon$.

Proof of Lemma 2. We write $S(u, w)=R([k]+1, w)-R([k]+1, u)$. Then by (3) and (2)(a), $-\Gamma(1-\langle k\rangle) S(u, w)$ equals

$$
\int_{u}^{w}(t-u)^{-\langle k\rangle} e^{t s^{\prime}} f(t) d t+\int_{w}^{\infty}\left\{(t-u)^{-\langle k\rangle}-(t-w)^{-\langle k\rangle}\right\} e^{t s^{\prime}} f(t) d t
$$

Now by an integration by parts, $e^{-w s^{\prime}} U^{(\langle k\rangle+\varepsilon)}(w)$ has value

$$
c_{1} e^{-w s^{\prime}} S(w-1, w)+c_{2} e^{-w s^{\prime}} \int_{w-1}^{w} S(u, w)(w-u)^{\langle k\rangle+\varepsilon-2} d u=G+H,
$$

say. By (2)(b), $G$ is in $V$. We now insert (8) in $H$, obtaining $H_{1}+H_{2}$, say. In $H_{1}$ we put $t=w-y$ and then $u=w-x$; in $H_{2}$ we put $t=w+v, u=w-x$. Applying [6, Lemma 2], we see that $H_{1}$ and $H_{2}$ are in $V$. This completes the proof.

3. The abscissa $\bar{\sigma}_{k}$. We shall write $\bar{\sigma}_{k}$ for the infimum of the set of $\sigma$ 's for which $C(w)$ is summable $|C, k|$, and $\bar{k}$ for the infimum of the set of $k$ 's for which $\bar{\sigma}_{k}$ is less than infinity. It is known (see [9], [7], [2, Lemma 13]) that $\bar{\sigma}_{k}$ is continuous for $k>k$, the value $-\infty$ being allowed. We have now, as a deduction from Theorems A and B (compare [4, pp. 470, 475]):

THEOREM A*. If $k$ is positive and fractional, $k>\bar{k}$ and $C(w)$ is summable $|C, k|$ for some $s$ such that $\sigma<0$, then $R(k, w)$ exists in the $|C, k|$ sense and $e^{-w s^{\prime}} R(k, w)=o(1)|C, 0|$ for $\sigma^{\prime}>\sigma$.

THEOREM $\mathrm{B}^{*}$. If $k$ is positive and fractional, $k>k$ and if $R(k, w)$ exists in the $(C, p)$ sense for some $p \geqq k$, and

(9) $e^{-w s^{\prime}} R(k, w)$ is summable $|C, 0|$ for some $s^{\prime}$ such that $\sigma^{\prime}<0$, then $C(w)$ is summable $|C, k|$ for every $\sigma>\sigma^{\prime}$. 
We observe that the conclusion of Theorem $\mathrm{A}^{*}$ implies

$$
\int_{w}^{\infty}\left|d_{w} R(k, w)\right|=O\left(e^{w \sigma^{\prime}}\right) \quad\left(\sigma^{\prime}>\sigma\right)
$$

and it is not difficult to see that (10), together with the condition

$$
R(k, w) \rightarrow 0 \text { as } w \rightarrow \infty
$$

implies the hypothesis (9) of Theorem B*, with a slightly larger $\sigma^{\prime}$. Since certainly $R(k, w)$ exists in the $(C, k)$ sense (by [1, p. 300]) and also (11) holds (by Theorem $\mathrm{A}^{*}$ ) if we assume that $\bar{\sigma}_{k}$ is negative, we obtain the following formula for $\bar{\sigma}_{k}$ in the case $k$ fractional (see [4, p. 463]), a similar argument yielding the case $k$ integral:

Theorem C. Let $\bar{\sigma}_{k}$ be negative. Then if $k=0,1, \cdots$, or if $k$ is fractional and $k>\bar{k}$,

$$
\bar{\sigma}_{k}=\limsup _{w \rightarrow \infty} w^{-1} \log \int_{w}^{\infty}|d R(k, u)| .
$$

If $k$ is fractional and $k=\bar{k}$, it is possible for the formula to fail.

For the second part we choose the function $A(u)$ used in the proof of $\left[6\right.$, Theorem $\left.A^{\prime \prime \prime}\right]$; then the right side of (12) is infinity.

\section{BIBLIOGRAPHY}

1. L. S. Bosanquet, The summability of Laplace-Stieltjes integrals, Proc. London Math. Soc. (3) 3 (1953), 267-304. MR 15, 307.

2. Soc. (3) 11 (1961), 654-690. MR 25 \#5352.

3. G. L. Isaacs, An extension of a limitation theorem of M. Riesz, J. London Math. Soc. 33 (1958), 406-418. MR 21 \#270.

4. - On the summability-abscissae of Laplace integrals, Proc. London Math. Soc. (3) 10 (1960), 461-479. MR 22 \#9815.

5. - The iteration formula for inverted fractional integrals, Proc. London Math. Soc. (3) 11 (1961), 213-238. MR 23 \#A1979.

6. - A limitation theorem for absolute summability, Proc. Amer. Math. Soc. 29 (1971), 47-54.

7. A. Peyerimhoff, Über einen absoluten Mittelwertsatz und Konvexitätssatz für Rieszsche Mittel, Math. Ann. 157 (1964), 42-64. MR 30 \#1337.

8. B. Prasad, Certain aspects of the researches on Dirichlet series, Twenty-Seventh Conference, Indian Mathematical Society, Ahmedabad, India, 1961.

9. P. Srivastava, On the abscissa of absolute summability of a Dirichlet series, Indian J. Math. 1 (1959), 77-86. MR 22 \#6977.

Department of Mathematics, Herbert H. Lehman College (CUNY), Bronx, NeW YORK 10468 